\title{
HU multimerization shift controls nucleoid compaction
}

\author{
Michal Hammel $^{1}$ \\ ${ }^{1}$ Lawrence Berkeley National Lab, Berkeley, United States \\ E-mail: mhammel@lbl.gov
}

Molecular mechanisms controlling functional bacterial chromosome (nucleoid) compaction and organization are surprisingly enigmatic, but depend partly upon conserved, histone-like proteins HUaa and Hua $\beta$ and their interactions that span the nano and meso scales from protein-DNA complexes to the bacterial chromosome and nucleoid structure. Here, we determined crystal structures of these chromosome-associated proteins in complex with native duplex DNA. Distinct DNAbinding modes of HUaa- and $\mathrm{HUa} \beta$ elucidate fundamental features of bacterial chromosome packing regulating gene transcription. By combining crystal structures with solution X-ray scattering results, we determined architectures of HU-DNA nucleoproteins in solution under near physiological conditions. These macromolecular conformations and interactions result in contraction at the cellular level based upon in vivo imaging of native unlabeled nucleoid by soft X-ray tomography upon HU $\beta$ and ectopic HUa38 expression. Structural characterization of charge-altered HUaa - DNA complex reveals an HU molecular switch suitable to condense nucleoid and reprogram noninvasive Escherichia coli into an invasive form. Collective findings suggest that shifts between networking, cooperative and non-cooperative DNA-dependent HU multimerization control DNA compaction and supercoiling independently of cellular topoisomerase activity. By integrating X-ray crystal structures, X-ray scattering, mutational tests, and X-ray imaging that span from protein-DNA complexes to the bacterial chromosome and nucleoid structure, we show that defined dynamic HU interaction networks can promote nucleoid reorganization and transcriptional regulation as efficient general microbial mechanisms to help synchronize genetic responses to cell cycle, changing environments, and pathogenesis.

Hammel, M., D. Amlanjyoti, F. E. Reyes, J. H. Chen, R. Parpana, H. Y. Tang, C. A. Larabell, J. A. Tainer and S. Adhya (2016). Sci Adv 2(7): e1600650.

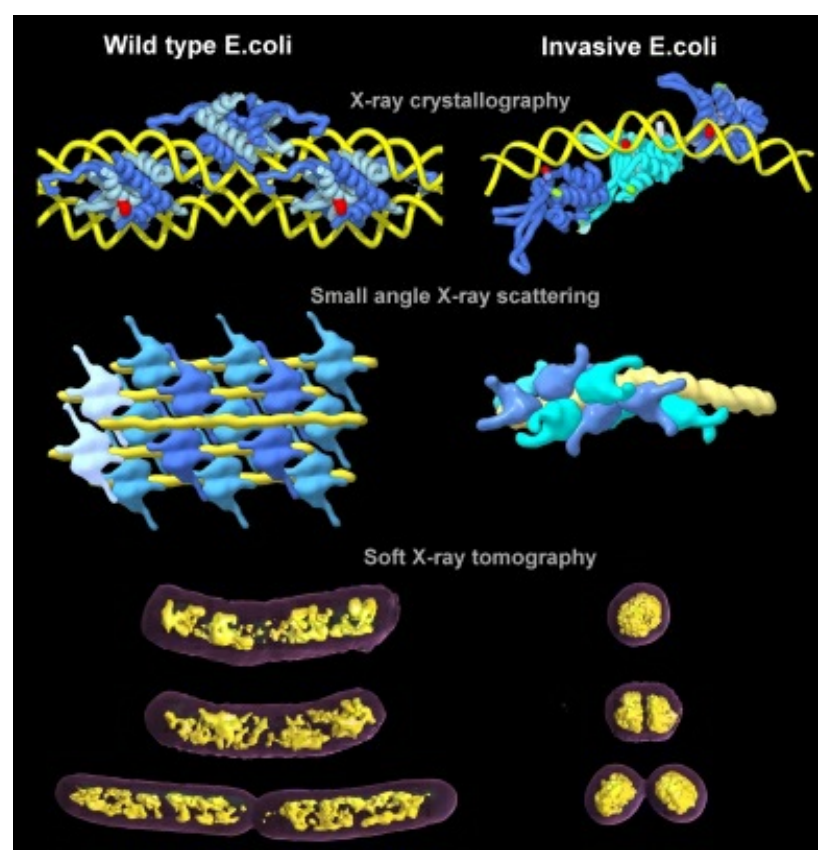

Keywords: $\underline{\text { HU-DNA structures, transcription, and pathogenicity }}$ 